\title{
Psychometric Properties of the Diabetes Management Self-Efficacy Scale in Korean Patients with Type 2 Diabetes
}

\author{
Eun-Hyun Lee, ${ }^{1}$ Jaap van der Bijl, ${ }^{2}$ Lillie M. Shortridge-Baggett, ${ }^{3}$ \\ Seung Jin Han, ${ }^{4}$ and Seung Hei Moon ${ }^{5}$ \\ ${ }^{1}$ Graduate School of Public Health, Ajou University, 164 Worldcup-ro, Yeongtong-gu, Suwon-si, \\ Gyeonggi-do 443-380, Republic of Korea \\ ${ }^{2}$ Inholland University of Applied Sciences, De Boelelaan 1109, 1081 HV Amsterdam, The Netherlands \\ ${ }^{3}$ Department of Graduate Studies, Lienhard School of Nursing, Pace University, 861 Bedford Road, Pleasantville, \\ NY 10570-2799, USA \\ ${ }^{4}$ Department of Endocrinology and Metabolism, School of Medicine, Ajou University, Suwon, \\ Gyeonggi-do 443-380, Republic of Korea \\ ${ }^{5}$ Department of Nursing, Graduate School, Inha University, 100 Inha-ro, Nam-gu, Incheon 402-751, Republic of Korea
}

Correspondence should be addressed to Eun-Hyun Lee; ehlee@ajou.ac.kr

Received 29 August 2014; Accepted 17 November 2014

Academic Editor: Anastasia Mavrogiannaki

Copyright (C) 2015 Eun-Hyun Lee et al. This is an open access article distributed under the Creative Commons Attribution License, which permits unrestricted use, distribution, and reproduction in any medium, provided the original work is properly cited.

\begin{abstract}
Objectives. The aims of this study were to perform a cultural translation of the DMSES and evaluate the psychometric properties of the translated scale in a Korean population with type 2 diabetics. Methods. This study was conducted in patients with diabetes recruited from university hospitals. The first stage of this study involved translating the DMSES into Korean using a forwardand backward-translation technique. The content validity was assessed by an expert group. In the second stage, the psychometric properties of the Korean version of the DMSES (K-DMSES) were evaluated. Results. The content validity of the K-DMSES was satisfactory. Sixteen-items clustered into four-subscales were extracted by exploratory factor analysis, and supported by confirmatory factor analysis. The construct validity of the K-DMSES with the Summary of Diabetes Self-Care Activities scale was satisfactory $(r=0.50, P<0.001)$. The Cronbach's alpha and intraclass correlation coefficient were 0.92 and $0.85(P<0.001 ; 95 \%$ $\mathrm{CI}=0.75-0.91)$, respectively, which indicate excellent internal consistency reliability and test-retest reliability. Conclusions. The K-DMSES is a brief instrument that has demonstrated good psychometric properties. It is therefore feasible to use in practice, and is ready for use in clinical research involving Korean patients with type 2 diabetes.
\end{abstract}

\section{Introduction}

The prevalence of diabetes has reached an almost epidemic level. About 382 million people in the world have diabetes, and this number is expected to rise to 592 million by 2035 [1]. The prevalence of diagnosed diabetes in Korea has increased from $2 \%$ in the 1970 s to $9.8 \%$ in 2011, and in 2012 the rate for patients with poor glycemic control was reportedly as high as $71.5 \%$ [2]. These findings suggest the presence of a substantial financial burden on the Korean health-care system.

Diabetes can be substantially improved by performing tasks such as taking prescribed medications, monitoring blood glucose levels, eating an appropriate diet, and exercising regularly. These are all day-to-day behaviors that patients must carry out to control their disease, a process that is termed self-management [3]. The traditional approach to diabetes self-management has been to educate patients about the disease and provide them with the skills necessary to control it [4]. According to one systematic review, although such self-management education appears to be successful, it exerts only small-to-moderate effects on the diabetes [5]. Diabetes researchers insist that providing patients with knowledge and skills is crucial, but these approaches appear to be insufficient for including the required behavioral changes 
among patients with diabetes $[6,7]$. Therefore, further factors that contribute to more effective diabetes self-management need to be considered.

Self-efficacy, a term that is derived from the social cognitive theory, refers to "belief in one's capability to organize and execute the course of action required to produce given levels of attainments" [8]. Self-efficacy influences the individual's choice of behaviors; people tend to engage in tasks when they feel competent to perform them and to avoid them when they feel that they exceed their capabilities. Self-efficacy also influences how people motivate themselves in the tasks that they undertake. That is, people with a strong sense of selfefficacy view their tasks or behaviors as challenges to be mastered, even if they are difficult. Efficacious people tend to set challenging goals and maintain commitment to them. In addition, self-efficacy beliefs influence emotional states; people with higher self-efficacy are likely to have reduced stress levels and lower risks of depression than those with low self-efficacy [9]. Thus, self-efficacy has emerged as a crucial factor in diabetes self-management behaviors [10-12].

Instruments that measure self-efficacy are broadly categorized into general and specific types of scales. Some researchers view self-efficacy as a more trait-like general construct, referring to one's overall competence to perform across a variety of different situations $[13,14]$. Instruments developed based on this perspective are general self-efficacy scales. Others state that self-efficacy judgments are specific to behaviors and the situations in which those behaviors occur $[15,16]$; that is, people perceive different levels of capability of performing in particular domains or situations of functioning. Instruments developed from this conceptualization are specific self-efficacy scales. Patients with diabetes must perform particular tasks to control their blood glucose in order to prevent complications. They may possess a high self-efficacy with respect to taking medication, but a low self-efficacy regarding physical exercise. Scales that are specifically designed for patients with diabetes are therefore more appropriate for measuring their self-efficacy $[10,17,18]$.

There have been previous attempts in Korea to develop a specific scale measuring the perceived self-efficacy of diabetes self-management $[19,20]$, but they have produced only a primitive stage of scale development; the items were derived from the literature without verifying their psychometric properties. Applying such instruments in the studies for clinical interventions may threaten the reliability of their outcomes. The Diabetes Management Self-Efficacy Scale (DMSES) is a specific-type instrument that was developed by the members of the International Partnership in SelfManagement and Empowerment [21]. Its psychometric properties were found to be acceptable for populations with type 2 diabetes in several countries: Netherlands [21], United Kingdom [22], Australia [23], Turkey [24], and Taiwan [25]. However, these psychometric studies had methodological and statistical problems related to factors such as sample size, item redundancy, and the underlying constructs. With these issues in mind, the aims of the present study were to perform a culture-sensitive translation of the DMSES and then evaluate the psychometric properties of the translated scale in a Korean population with type 2 diabetes.

\section{Methods}

2.1. Step I: Cultural Translation and Content Validity. The English-language version of the DMSES was translated into Korean using a forward and backward translation technique, based on the guidelines of Brislin [26]. A bilingual health professional and a layperson independently translated the English version into Korean using semantic equivalence. An expert panel of three bilinguals checked the two potential Korean versions and achieved a consensus on a Korean version. The Korean version was then independently translated back into English by another two bilinguals. The panel checked the back-translated versions against the original English version. Any discrepancies between the translated and original English versions were either confirmed by one of the original developers or else a consensus was reached by the panel. The preliminary Korean version was thus produced, and the Korean version was finalized after one professor majored in Korean literature had reviewed its grammar.

Five experts (one physician, one professor in nursing, and three diabetes educators) were involved in assessing the content validity of the final Korean version of the DMSES (KDMSES). These experts were asked to rate each item of the preliminary K-DMSES whether they considered it "essential," "useful, but not essential," or "not essential" [27]. In addition, they were asked to answer open questions regarding whether or not there were any ambiguous words, jargon, or valueladen words and whether or not there were items that needed to be modified.

\subsection{Step II: Psychometric Evaluation of the K-DMSES}

2.2.1. Participants and Procedures. This was a methodological study to assess the psychometric properties of the K-DMSES. A convenience sample of 440 patients with type 2 diabetes was recruited from two university hospitals in South Korea. This sample size satisfied the requirement that at least 7 times the total number of items is needed for psychometric tests [28]. The inclusion criteria for the participants were being aged at least 20 years, being diagnosed with diabetes type 2, and being articulate in the Korean language. The participants were asked to sign a consent form and complete a package of questionnaires. Of these, 70 were given an envelope containing the K-DMSES questionnaire for the assessment of test-retest reliability. They were asked to take it home and complete it 10 days later; a time interval of 1-2 weeks between repeated measures is often recommended [28]. Each participant was asked to post the return envelope containing the completed questionnaire near home.

2.2.2. Ethical Consideration. Prior to data collection, this study was approved by the institutional review boards at the participating institutions. Participants were voluntary and those who agreed to participate signed a consent form. All participants were assured of their confidentiality.

2.2.3. Questionnaires. The DMSES [21] is a self-reported questionnaire that comprises 20 items with 4 subscales: nutrition specific and weight, nutrition general and medical 
treatment, physical exercise, and blood sugar. Originally, each item was scored on a 5 -point scale, but this was later revised to an 11-point scale on the UK English-language version [22]. Possible scores range from 0 to 200, with higher scores reflecting higher self-efficacy. The DMSES satisfied the content validity, factorial construct validity, internal consistency reliability, and test-retest reliability when it was developed. The English-language version of the DMSES, which was obtained from the developer, was translated into Korean and used in this study.

Based on previous studies [12, 25], it was hypothesized in this study that the DMSES was positively and moderately correlated with the Summary of Diabetes Self-Care Activities Scale (SDSCA) [29]. Therefore, the Korean version of the SDSCA was administered to test hypothesis testing construct validity. The SDSCA assesses the frequency of behavioral tasks in five aspects of the diabetes regimen: diet, exercise, self-monitoring of blood glucose, foot care, and smoking for the previous 7 days. The reliability and validity of the SDSCA, which comprises 11 items, were culturally adapted for Korean patients with type 2 diabetes [30, 31].

2.2.4. Statistical Analyses. Statistical analyses were completed using the PASW (version 18) statistical package. General characteristics and missing data were calculated using descriptive statistics. The zero-order correlation matrix among the K-DMSES items was computed using Pearson's analysis.

A cross-validation approach involving both exploratory factor analysis (EFA) and confirmatory factor analysis (CFA) was used for the factorial construct analysis, and for the cross-validation, 440 patients were split into 2 subsamples using a random-sampling function of the computer program (Table 1). The homogeneity of the subsamples with regard to general characteristics was computed using $\chi^{2}$ or Fisher's exact test. With subsample 1, Bartlett's test of sphericity and the Kaiser-Meyer-Olkin (KMO) measure of sampling adequacy were screened to justify undertaking EFA [32]. Then, EFA was performed using principal-axis factor analysis with Varimax rotation. Factors with an eigenvalue higher than 1 were retained, and the factor loading criterion was set at $\geq 0.4$ [33]. For the CFA with subsample 2, a maximum-likelihood estimation procedure was performed. Multiple criteria were used to evaluate the model fit: the ratio of the $\chi^{2}$ value to the degrees of freedom (CMIN/DF), goodness-of-fit index (GFI), standardized root-mean-square residual (SRMR), root-mean-square error of approximation (RMSEA), comparative fit index (CFI), and normed fit index (NFI). The following criteria were used to confirm that a model was an acceptable fit: relative CMIN/DF $<3$, GFI > 0.9, SRMR $<0.08$, RMSEA $<0.08$, CFI $>0.9$, and NFI $>0.9$ [33-36].

Construct validity by means of the hypothesis testing approach was examined for the entire sample using Pearson's correlation analysis. Internal consistency reliability and testretest reliability were evaluated using Cronbach's alpha and the intraclass correlation coefficient (ICC), respectively.

\section{Results}

3.1. Step 1: Cultural Translation and Content Validity. In Korean culture, workers often go out after work to socialize, either formally or informally, as a release from their jobrelated stresses, and this socializing often involves eating grilled meats or rice and drinking alcohol. It is difficult for a worker at a group dinner to refuse to eat or drink or to order other foods for only himself/herself. Thus, the term "company dinner" was added in the translation process to item 16: "... able to follow a healthy eating pattern when I am eating out, at a party, or at a company dinner." Clinicians in Korea usually recommend that patients with diabetes visit their physicians every 3 months, based on the guidelines of the Korean Diabetes Association [37]. Therefore, item 18 (“... able to visit my doctor once a year to monitor my diabetes") was changed to "... able to visit a clinic or a public health center four times a year to monitor my diabetes."

With respect to the content validity, all of the experts considered all of the items to be essential. However, item 11 ("... able to exercise more if the doctor advises me to") was refined by replacing the term "doctor" in this item with "health professional," since patients with diabetes in Korea receive advice not only from physicians but also from diabetes educators (e.g., nurses or nutritionists). Three experts commented that there were content similarities between items 4 and 5 and between items 13 and 14; however, no deletions were performed at this stage. The experts recommended additional quantitative analysis. All 20 items were retained for the next step of psychometric evaluation.

\subsection{Step 2: Psychometric Evaluation of the K-DMSES}

3.2.1. Missing Data. The rate of missing values was $0.23 \%$ for each of items 3,11, and 15; these missing values were replaced by the mean value for each item. There were no missing values for any of the other items.

3.2.2. Zero-Order Correlation Matrix. In the $20 \times 20$ zeroorder correlation matrix, items $4 / 5,14 / 13$, and $16 / 15$ were strongly correlated $(r=0.80-0.90)$, as expected from the results for content validity. These strong correlations indicate the presence of redundancy [38], and hence only one item of each pair was retained. Items 4 and 14 were retained because their contents are more specific to diabetes than those of items 5 and 13. Furthermore, item 16 ("eat out, at a party, or at a company dinner") occurs more frequently in daily life than the content of item 15 ("eat on holiday"), and so item 16 was retained. Thus, items 5,13 , and 15 were deleted in order to remove content redundancy.

3.2.3. Factorial Construct Validity. The general characteristics did not differ between subsamples, as assessed by $\chi^{2}$ or Fisher's exact test (Table 1). With the randomly split subsample 1 , the KMO statistic (0.89) and Bartlett's sphericity $\left(\chi^{2}=\right.$ 2602.62, $P<0.001)$ indicated that the correlation matrix was suitable for factor analysis. The initial EFA extracted a fourfactor solution (eigenvalue $>1$, Table 2), which accounted 
TABLE 1: General characteristics.

\begin{tabular}{|c|c|c|c|}
\hline Variable & $\begin{array}{c}\text { Subsample } 1 \\
(n=220) \\
n(\%)\end{array}$ & $\begin{array}{c}\text { Subsample } 2 \\
(n=220) \\
n(\%)\end{array}$ & $\begin{array}{c}\chi^{2} \text { or Fisher's exact } \\
\text { test }(P)\end{array}$ \\
\hline Gender & & & $0.146(0.703)$ \\
\hline Male & $111(50.5)$ & $115(52.3)$ & \\
\hline Female & $109(49.5)$ & $105(47.7)$ & \\
\hline Age (years) $($ mean $\pm S D=58.02 \pm 0.88)$ & & & $3.902(0.561)$ \\
\hline $20-29$ & $2(0.9)$ & $3(1.4)$ & \\
\hline $30-39$ & $7(3.2)$ & $10(4.5)$ & \\
\hline $40-49$ & $34(15.5)$ & $30(13.6)$ & \\
\hline $50-59$ & $87(39.5)$ & $71(32.3)$ & \\
\hline $60-69$ & $57(25.9)$ & $68(30.9)$ & \\
\hline$\geq 70$ & $33(15.0)$ & $38(17.3)$ & \\
\hline Marital status & & & $0.705(0.894)$ \\
\hline Married/living together & $178(80.9)$ & $173(78.6)$ & \\
\hline Divorced/widow(er) & $32(14.5)$ & $37(16.8)$ & \\
\hline Unmarried & $9(4.1)$ & $9(4.1)$ & \\
\hline Other & $1(0.5)$ & $1(0.5)$ & \\
\hline Job & & & $2.142(0.295)$ \\
\hline Employed & $102(46.4)$ & $114(51.8)$ & \\
\hline None & $117(53.1)$ & $106(48.2)$ & \\
\hline Data missing & $1(0.5)$ & $0(0.0)$ & \\
\hline Education & & & $1.230(0.873)$ \\
\hline Elementary school & $34(15.5)$ & $35(15.9)$ & \\
\hline Middle school & $30(13.6)$ & $32(14.5)$ & \\
\hline High school & $88(40.0)$ & $88(40.0)$ & \\
\hline College and above & $59(26.8)$ & $60(27.3)$ & \\
\hline Other & $9(4.1)$ & $5(2.3)$ & \\
\hline Monthly income (KRW) & & & $1.147(0.766)$ \\
\hline Less than $2,000,000$ & $88(40.0)$ & $79(35.9)$ & \\
\hline $2,000,000-2,999,999$ & $40(18.2)$ & $39(17.7)$ & \\
\hline $3,000,000-3,999,999$ & $33(15.0)$ & $40(18.2)$ & \\
\hline $4,000,000$ and above & $56(25.4)$ & $54(24.5)$ & \\
\hline Data missing & $3(1.4)$ & $8(3.6)$ & \\
\hline Treatment regimen & & & $1.164(0.762)$ \\
\hline Diet/exercise only & $9(4.1)$ & $7(3.2)$ & \\
\hline Oral hypoglycemic agent & $141(64.1)$ & $151(68.6)$ & \\
\hline Insulin & $10(4.5)$ & $10(4.5)$ & \\
\hline Oral hypoglycemic agent + insulin & $60(27.3)$ & $52(23.6)$ & \\
\hline $\operatorname{HbAlc}($ mean $\pm \mathrm{SD}=7.70 \pm 1.38)$ & & & $0.011(0.918)$ \\
\hline Controlled $(\mathrm{HbAlc}<7.0 \%)$ & $69(31.4)$ & $70(31.8)$ & \\
\hline Uncontrolled (HbAlc $\geq 7.0 \%)$ & $151(68.6)$ & $150(68.2)$ & \\
\hline
\end{tabular}

HbAlc: hemoglobin Alc; KRW: South Korean won.

for $65.81 \%$ of the total variance. Item 7 was not significantly loaded on any factors at a criterion of $>0.40$. EFA was conducted after deleting that item (Table 2 ), again yielding a four-factor solution that explained $67.28 \%$ of the total variance in all items. All items were significantly loaded onto one of four factors. There was no significant cross-loading of items on the factors. Factors 1-4 were labeled "nutrition" (items 4, 9, 10, 14, 16, and 17), "physical exercise/body weight" (items 6, 8, 11, and 12), "medical treatment" (items 18, 19, and 20), and "blood sugar" (items 1, 2, and 3).

To cross-validate the 16-item, 4-factor construct, CFA was conducted with the randomly split subsample 2 . The 
TABLE 2: Exploratory factor analyses.

\begin{tabular}{|c|c|c|c|c|c|c|c|c|c|}
\hline \multirow{2}{*}{ Number } & \multirow{2}{*}{$\begin{array}{l}\text { Abbreviated item } \\
\text { description }\end{array}$} & \multicolumn{4}{|c|}{ First exploratory factor analysis ${ }^{\mathrm{a}}$} & \multicolumn{4}{|c|}{ Second exploratory factor analysis ${ }^{\mathrm{b}}$} \\
\hline & & $\mathrm{F}^{\mathrm{c}}$ & $\mathrm{F} 2^{\mathrm{d}}$ & $\mathrm{F}^{\mathrm{e}}$ & $\mathrm{F} 4^{\mathrm{f}}$ & $\mathrm{Fl}^{\mathrm{c}}$ & $\mathrm{F} 2^{\mathrm{d}}$ & $\mathrm{F} 3^{\mathrm{e}}$ & $F 4^{\mathrm{f}}$ \\
\hline 1 & Checking blood sugar & & & & 0.46 & & & & 0.46 \\
\hline 2 & $\begin{array}{l}\text { Correcting high blood } \\
\text { sugar }\end{array}$ & & & & 0.80 & & & & 0.84 \\
\hline 3 & Correcting low blood sugar & & & & 0.83 & & & & 0.81 \\
\hline 4 & Choosing foods & 0.52 & & & & 0.53 & & & \\
\hline 6 & Controlling body weight & & 0.59 & & & & 0.58 & & \\
\hline 7 & Examining feet for cuts & & & & & - & - & - & - \\
\hline 8 & Taking physical exercise & & 0.74 & & & & 0.74 & & \\
\hline 9 & $\begin{array}{l}\text { Adjusting eating plan } \\
\text { during illness }\end{array}$ & 0.53 & & & & 0.54 & & & \\
\hline 10 & $\begin{array}{l}\text { Following a healthy eating } \\
\text { pattern }\end{array}$ & 0.67 & & & & 0.57 & & & \\
\hline 11 & $\begin{array}{l}\text { Taking physical exercise on } \\
\text { doctor's advice }\end{array}$ & & 0.84 & & & & 0.81 & & \\
\hline 12 & $\begin{array}{l}\text { Balancing between exercise } \\
\text { and eating plan }\end{array}$ & & 0.74 & & & & 0.74 & & \\
\hline 14 & $\begin{array}{l}\text { Adjusting eating plan: } \\
\text { when I am away from home }\end{array}$ & 0.75 & & & & 0.76 & & & \\
\hline 16 & $\begin{array}{l}\text { Eating pattern: eating out, } \\
\text { eating at a party or } \\
\text { company dinner }\end{array}$ & 0.80 & & & & 0.79 & & & \\
\hline 17 & $\begin{array}{l}\text { Eating plan related to stress } \\
\text { or anxiety }\end{array}$ & 0.76 & & & & 0.74 & & & \\
\hline 18 & $\begin{array}{l}\text { Visiting doctor four times a } \\
\text { year }\end{array}$ & & & 0.73 & & & & 0.70 & \\
\hline 19 & $\begin{array}{l}\text { Taking medication as } \\
\text { prescribed }\end{array}$ & & & 0.87 & & & & 0.91 & \\
\hline 20 & $\begin{array}{l}\text { Adjusting medication } \\
\text { during illness }\end{array}$ & & & 0.74 & & & & 0.73 & \\
\hline
\end{tabular}

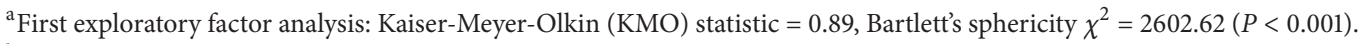

${ }^{\mathrm{b}}$ Second exploratory factor analysis: KMO statistic $=0.89$, Bartlett's sphericity $\chi^{2}=2461.10(P<0.001)$.

${ }^{\mathrm{c}}$ Factor 1: nutrition.

${ }^{\mathrm{d}}$ Factor 2: physical exercise/body weight.

${ }^{\mathrm{e}}$ Factor 3: medical treatment.

${ }^{\mathrm{f}}$ Factor 4: blood sugar.

TABLE 3: Summary of fit indices from confirmatory factor analysis.

\begin{tabular}{lcccccccc}
\hline & $\chi^{2}$ & df & CMIN/DF & GFI & SRMR & RMSEA (90\% CI) & CFI & NFI \\
\hline Model 1 & $391.57^{*}$ & 98 & 3.99 & 0.81 & 0.07 & $0.12(0.10-0.13)$ & 0.87 & 0.84 \\
Model 2 & $325.06^{*}$ & 97 & 3.35 & 0.85 & 0.06 & $0.10(0.09-0.11)$ & 0.90 & 0.97 \\
Model 3 & $284.50^{*}$ & 96 & 2.96 & 0.87 & 0.06 & $0.09(0.08-0.10)$ & 0.92 & 0.88 \\
Model 4 & $253.11^{*}$ & 95 & 2.66 & 0.88 & 0.06 & $0.08(0.07-0.10)$ & 0.93 & 0.90 \\
\hline
\end{tabular}

df: degrees of freedom; CMIN/DF: ratio of $\chi^{2}$ value to the degrees of freedom; GFI: goodness-of-fit index; SRMR: standardized root-mean-square residual; RMSEA ( $90 \%$ CI): root-mean-square error of approximation with $90 \%$ of confidence interval; CFI: comparative fit index; NFI: normed fit index.

${ }^{*} P<0.001$.

SRMR value indicated an acceptable model fit, where the values of the other indexes indicated a poor-fitting model (Model 1, Table 3). Thus, the possibility of model modification was explored using modification indices (MIs) [39], which revealed that the MI value of pairing of error terms between items 14 and 16 was the largest, at 57.38. After modifying the covariance between the error terms of items 14 and 16 (Model $2)$, the model fit was significantly improved $\left(\Delta \chi^{2}(1)=66.51\right.$, $P<0.05)$. However, the values of some model-fit indexes (CMIN/DF, GFI, and RMSEA) were unsatisfactory, and there was still a large MI value (36.63) between the error terms of items 16 and 17. With this modification, CFA produced a 


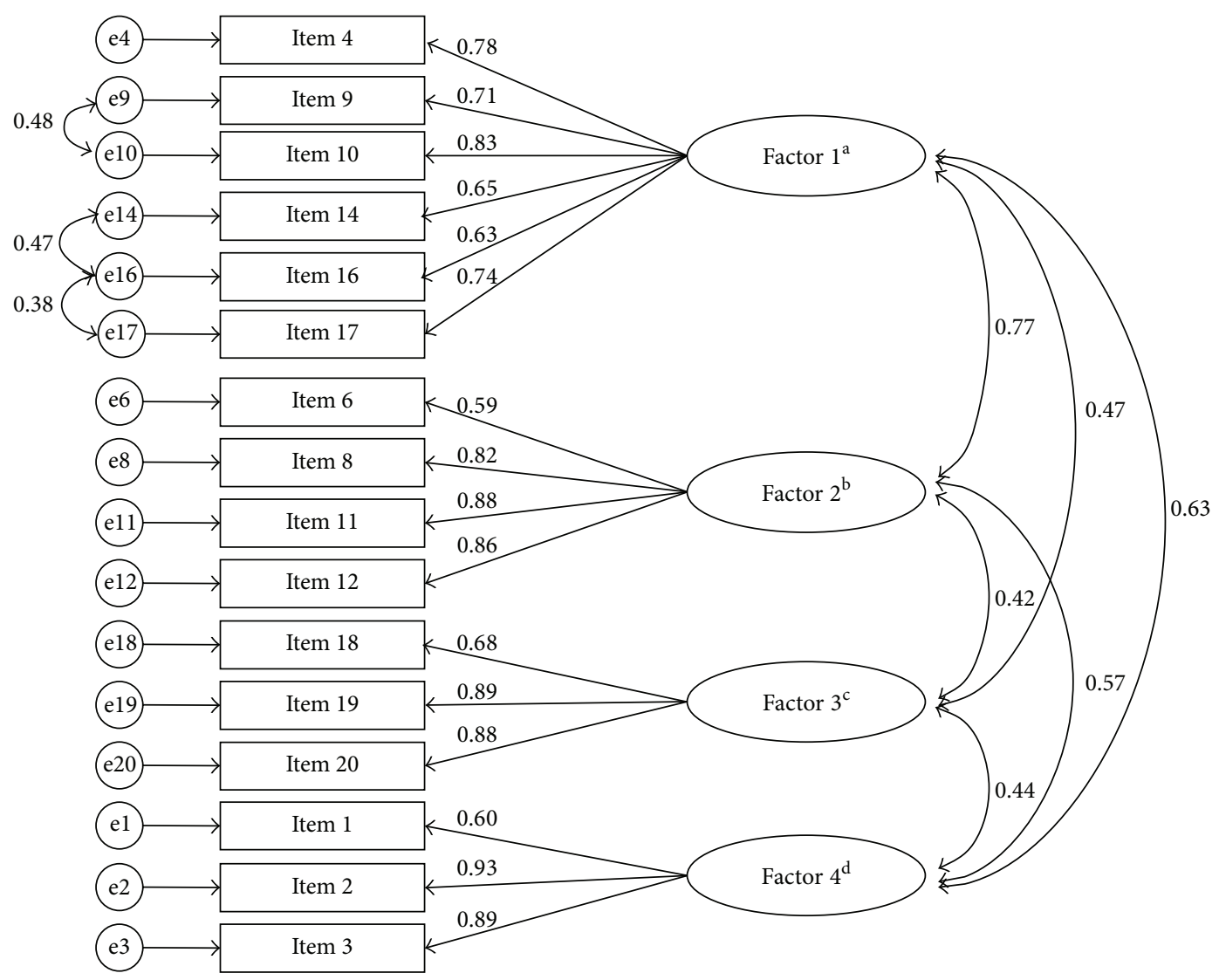

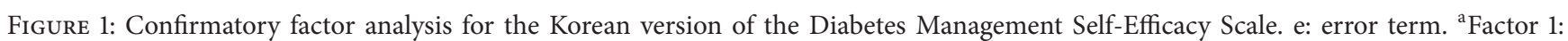
nutrition. ${ }^{\mathrm{b}}$ Factor 2: physical exercise/body weight. ${ }^{\mathrm{c}}$ Factor 3: medical treatment. ${ }^{\mathrm{d}}$ Factor 4: blood sugar.

significantly improved Model $3\left(\Delta \chi^{2}(1)=40.56, P<0.05\right)$. After the final modification of the covariance between the error terms of items 9 and $10(\mathrm{MI}=17.06)$, Model 4 was significantly improved compared with Model $3\left(\Delta \chi^{2}(1)=\right.$ $31.39, P<0.05)$, and the values of all goodness-of-fit indexes, except GFI, were satisfactory. All items loaded meaningfully onto factors with standardized values ranged from 0.59 to 0.93 (Figure 1).

3.2.4. Hypothesis Testing Construct Validity with the Total Sample. The K-DMSES score was moderately correlated with the SDSCA score $(r=0.50, P<0.001)$, as hypothesized for the construct validity.

\subsubsection{Internal Consistency Reliability with the Total Sample.} Overall Cronbach's alpha of the K-DMSES was 0.92, which indicates excellent internal consistency reliability. Cronbach's alpha values for the subscales of nutrition, physical exercise/body weight, medical treatment, and blood sugar were $0.89,0.87,0.86$, and 0.84 , respectively, which were all above the acceptability criterion of $\geq 0.70[40]$.

3.2.6. Test-Retest Reliability. Of the 70 patients who were asked to complete the K-DMSES twice, $82.85 \%(n=58)$ completed it twice. The ICC for the overall K-DMSES score was $0.85(P<0.001 ; 95 \%$ confidence interval $=0.75-0.91)$, reflecting a satisfactory test-retest reliability. ICCs for the nutrition, physical exercise/body weight, medical treatment, and blood sugar subscales were $0.87,0.78,0.62$, and 0.88 , respectively.

\section{Discussion}

This study translated the DMSES into Korean and evaluated its psychometric properties in Korean type 2 diabetes patients. The psychometric properties of the culturally adapted K-DMSES were satisfactory. The total number of items in the K-DMSES was 16, which is fewer than in all other language versions of the DMSES except for the UKEnglish version, which comprises 15 items [22]. A shorter KDMSES may represent a smaller burden for patients with type 2 diabetes, rendering it more feasible to use in practice.

Translation and back-translation of a questionnaire requires not only literal translation but also social/cultural adaptation. In this study, item 18 of the K-DMSES was changed to "... four times a year to monitor my diabetes," based on the guidelines of the Korean Diabetes Association. A similar change was also made in the Taiwanese/Chinese version [25], in accordance with Taiwanese regulations of the Bureau of National Health Insurance. In the UK version, the item was deleted based on the National Health Service (such 
as GP care system in the UK) [22]. The inclusion or wording of item 18 may depend upon the prevailing health system or health policy in the country in which the questionnaire will be used.

Item redundancy on the DMSES is constantly being discussed. McDowell et al. [23] reported strongly correlated items (items 2/3, 8/11,13/14,13/15, and 14/15) in the AustralianEnglish version. Sturt et al. [22] also noted duplicated items (items 4/5, 5/10,13/14, and 13/15) in the content validity of the UK-English version. Similarly, redundancy of items $4 / 5$, $13 / 14$, and $15 / 16$ was found in the K-DMSES for the content validity and the zero-order correlation matrix of items. If items of a scale are strongly correlated, it is recommended that the redundant ones should be dropped. This prevents a methodological problem with multicollinearity [38].

Factorial construct validity in this study demonstrated that the K-DMSES comprises four subscales. The items clustered into each subscale were similar to those of the Taiwanese/Chinese version [25]. The Dutch version also comprises four subscales, wherein the clustered items on the physical exercise and blood sugar subscales were similar to those of the two aforementioned versions, but the items on the other two subscales ("nutrition specific and weight" and "nutrition general and medical treatment") were clustered differently [21]. This finding in the study of the Dutch version may be attributable to the use of an insufficient sample size $(N=94)$ for a principal component analysis. An inadequate sample size was also a weakness in the psychometric study of the Turkish version of the DMSES $(N=101)$, which revealed three subscales [24]. In addition, a single subscale was reported for the UK-English version [22], which accounted for only $41 \%$ of total variance of all items. This unidimensionality is not congruent with the assertion that diabetes management of self-efficacy is multifaceted [41]. Moreover, the total amount of variance accounted for by that unidimensionality did not meet the criterion of $>50 \%$ [28].

Item 7 ("I am able to examine my feet for cuts") has been inconsistent in its loading on factor analyses: it loaded onto the general nutrition and medical treatment subscale of the Dutch version [21], the diet/feet control subscale of the Turkish version [24], and the blood sugar/feet check subscale of the Taiwanese/Chinese version [25]. Furthermore, the item was statistically deleted in the present study. This lack of consistency may be due to there being only one item related to the confidence of foot care in the DMSES, with this item possibly being treated as relatively heterogeneous, resulting in it being statistically clustered onto various subscales, or even deleted from the scale. If there were more items related to item 7 , its own subscale might have been constructed. Given that at least three items are required for a latent construct [42], it is recommended that two items should be added in future studies, for example, "confident of protecting my feet from hot and cold" and "confident of putting on shoes and socks at all times."

Only EFA has been performed to evaluate the factorial construct validity of the DMSES-CFA has never been performed. This is the first study in which both EFA and CFA have been performed to validate the DMSES, applying a cross-validation approach. This approach has the merit of exploring the underlying construct of the items and simultaneously confirming the stability of those underlying constructs [43]. In the present study the four-subscale construct extracted from the EFA was empirically supported by CFA. However, the CFA revealed that there was covariance between the error terms of three pairs of items, items $9 / 10,14 / 16$, and $16 / 17$, implying the presence of an unknown systematic error. Byrne [39] reported that a systematic error may occur due to an overlap in the content of items. The contents of the three pairs of items all related to "eating-related confidence." Therefore, further study is needed to remove the possibility of content overlap.

Construct validation by means of the hypothesis testing approach refers to the correlation with one or more wellestablished instruments, based on a prior hypothesis [44]. The present study has demonstrated the construct validity of the K-DMSES, with a moderate correlation with the SDSCA. The Taiwanese/Chinese version of the DMSES exhibited a similar correlation $(r=0.58)$ to the SDSCA [25].

A Cronbach's alpha value of between 0.70 and 0.95 indicates sufficient item correlations and a low redundancy of items [28]. Overall Cronbach's alpha was a little higher for the K-DMSES (0.92) than for the Dutch version (0.81) [21], the UK-English version (0.89) [22], and the Turkish version (0.88) [24] and was similar to that of the AustralianEnglish version (0.91) [22] and the Chinese version (0.93) [25]. Together these findings suggest that the DMSES has a good internal consistency across languages.

Test-retest reliability refers to the temporal stability of a scale between two time points, and the most commonly used criteria for evaluating this parameter are Pearson's $r$ or ICC $>$ 0.70 [40]. Pearson's $r$ for the test-retest reliability ranged from 0.76 to 0.86 for the Dutch [21], Australian-English [22], and Chinese [25] versions. However, Pearson's $r$ is criticized for being insufficiently rigorous for assessing reliability. It does not consider systematic differences as a part of measurement error, so Pearson's $r$ value is usually higher than the ICC. The ICC is considered a more reliable parameter for continuous variables [28] and so was calculated in the present study, yielding a value of 0.85 , which is higher than that of the UK-English version (0.77) [22] and lower than that of the Turkish version (0.91) [24]. These findings suggest that the overall test-retest reliability of the DMSES is stable over time across languages. However, the medical treatment subscale in this study was characterized by a relatively low ICC (0.62). Similarly, the temporal stability of that subscale was unsatisfactory in the Taiwanese/Chinese version $(r=0.69)$ [25]. Other studies have determined only overall values, not values for the subscales, so it is currently difficult to determine why the medical treatment subscale lacks stability.

A limitation of this study is the lack of a responsiveness test to detect changes when patients improve or deteriorate [45]. A longitudinal study should therefore be conducted which assesses the K-DMSES scores of patients in whom changes are expected to occur.

Regarding test-retest reliability, the time interval between repeated measures should be justified. In general, it is preferable for the time interval to be sufficiently long to prevent recall, but short enough so as to ensure that a clinical 
change has not occurred [28]. Diverse time intervals have been applied in reliability testing of the DMSES: 10 days (present study), 2 weeks [25], 3 weeks [23], 4 weeks [22, 24], and 5 weeks [21]. One empirical study found no significant differences in the test-retest reliability of health-status instruments when time intervals of 2 days and 2 weeks were applied [46]. More studies of the optimal time interval for the testretest reliability of the DMSES are required.

\section{Conclusion}

The K-DMSES was subjected to culture-sensitive translation and its psychometric properties were validated in Korean type 2 diabetes patients. The underlying construct of the KDMSES comprises four subscales: nutrition (items 4, 9, 10, 14, 16, and 17), physical exercise/body weight (items 6,8 , 11 , and 12), medical treatment (items 18, 19, and 20), and blood sugar (items 1, 2, and 3). The K-DMSES demonstrated good content validity, factorial construct validity, hypothesis testing construct validity, internal consistency reliability, and test-retest reliability. This instrument is ready for use in both research and practice.

\section{Conflict of Interests}

The authors declare that there is no conflict of interests regarding the publication of this paper.

\section{Acknowledgment}

This research was supported by the Basic Science Research Program through the National Research Foundation of Korea (NRF) funded by the Ministry of Education, Science, and Technology (Grant no. 2012R1A1B5000978). The funder did not play any role in the conduct or publication of the study.

\section{References}

[1] International Diabetes Federation, Diabetes Atlas, 6th edition, 2013, http://www.idf.org/diabetesatlas/introduction.

[2] Korea Centers for Disease Control and Prevention, Korea Health Statistics 2011: Korea National Health and Nutrition Examination Survey (KNHANES V-2), Korea Center for Disease Control and Prevention \& Ministry of Health and Welfare, Seoul, Republic of Korea, 2011.

[3] N. M. Clark, M. H. Becker, N. K. Janz, K. Lorig, W. Rakowski, and L. Anderson, "Self-management of chronic disease by older adults: a review and questions for research," Journal of Aging and Health, vol. 3, no. 1, pp. 3-27, 1991.

[4] K. Krichbaum, V. Aarestad, and M. Buethe, "Exploring the connection between self-efficacy and effective diabetes selfmanagement," Diabetes Educator, vol. 29, no. 4, pp. 653-662, 2003.

[5] A. Warsi, P. S. Wang, M. P. LaValley, J. Avorn, and D. H. Solomon, "Self-management education programs in chronic disease: a systematic review and methodological critique of the literature," Archives of Internal Medicine, vol. 164, no. 15, pp. 1641-1649, 2004.
[6] R. E. Glasgow and V. L. Osteen, "Evaluating diabetes education: are we measuring the most important outcomes?" Diabetes Care, vol. 15, no. 10, pp. 1423-1432, 1992.

[7] K. M. Knight, T. Dornan, and C. Bundy, "The diabetes educator: trying hard, but must concentrate more on behaviour," Diabetic Medicine, vol. 23, no. 5, pp. 485-501, 2006.

[8] A. Bandura, "Health promotion from the perspective of social cognitive theory," Psychology and Health, vol. 13, no. 4, pp. 623649, 1998.

[9] A. Bandura, "Health promotion by social cognitive means," Health Education and Behavior, vol. 31, no. 2, pp. 143-164, 2004.

[10] A. C. Hurley and C. A. Shea, "Self-efficacy: strategy for enhancing diabetes self-care," The Diabetes Educator, vol. 18, no. 2, pp. 146-150, 1992.

[11] K. M. Nelson, L. McFarland, and G. Reiber, "Factors influencing disease self-management among veterans with diabetes and poor glycemic control," Journal of General Internal Medicine, vol. 22, no. 4, pp. 442-447, 2007.

[12] K. E. Williams and M. J. Bond, "The roles of self-efficacy, outcome expectancies and social support in the self-care behaviours of diabetics," Psychology, Health and Medicine, vol. 7, no. 2, pp. 127-141, 2002.

[13] M. Sherer, J. E. Maddux, B. Mercandante, S. Prentice-Dunn, B. Jacobs, and R. W. Rogers, "The self-efficacy scale: construction and validation," Psychological Reports, vol. 51, no. 2, pp. 663-671, 1982.

[14] J. X. Zhang and R. Schwarzer, "Measuring optimistic selfbeliefs: a Chinese adaptation of the general self-efficacy scale," Psychologia, vol. 38, pp. 174-181, 1995.

[15] A. Bandura, "Guide for constructing self-efficacy scales," in SelfEfficacy Beliefs of Adolescent, F. Pajares and T. Urban, Eds., pp. 307-337, Information Age Publishing, Charlotte, NC, USA, 2006.

[16] E. Maibach and D. A. Murphy, "Self-efficacy in health promotion research and practice: conceptualization and measurement," Health Education Research, vol. 10, no. 1, pp. 37-50, 1995.

[17] O. A. Al-Khawaldeh, M. A. Al-Hassan, and E. S. Froelicher, "Self-efficacy, self-management, and glycemic control in adults with type 2 diabetes mellitus," Journal of Diabetes and its Complications, vol. 26, no. 1, pp. 10-16, 2012.

[18] J. J. van der Bijl and L. M. Shortridge-Baggett, "The theory and measurement of the self-efficacy construct," Research and Theory for Nursing Practice, vol. 15, no. 3, pp. 189-207, 2001.

[19] J. H. Kim and S. A. Chang, "Effect of diabetes education program on glycemic control and self management for patients with type 2 diabetes mellitus," Korean Diabetes Journal, vol. 33, pp. 518-525, 2009.

[20] K. S. Paek and Y. H. Choi, "Sick-role behavioral compliance and influencing factors in patients with diabetes," Korean Journal of Adult Nursing, vol. 10, pp. 480-491, 1998.

[21] J. J. Van Der Bijl, A. Van Poelgeest-Eeltink, and L. ShortridgeBaggett, "The psychometric properties of the diabetes management self-efficacy scale for patients with type 2 diabetes mellitus," Journal of Advanced Nursing, vol. 30, no. 2, pp. 352359, 1999.

[22] J. Sturt, H. Hearnshaw, and M. Wakelin, "Validation and reliability of the DMSES UK: a measure of self-efficacy for type 2 diabetes self-management," Primary Health Care Research \& Development, vol. 11, pp. 374-381, 2010.

[23] J. McDowell, M. Courtney, H. Edwards, and L. ShortridgeBaggett, "Validation of the australian/english version of the 
diabetes management self-efficacy scale," International Journal of Nursing Practice, vol. 11, no. 4, pp. 177-184, 2005.

[24] M. Kara, J. J. van der Bijl, L. M. Shortridge-Baggett, T. Asti, and S. Erguney, "Cross-cultural adaptation of the diabetes management self-efficacy scale for patients with type 2 diabetes mellitus: scale development," International Journal of Nursing Studies, vol. 43, no. 5, pp. 611-621, 2006.

[25] S.-F. Vivienne Wu, M. Courtney, H. Edwards, J. McDowell, L. M. Shortridge-Baggett, and P.-J. Chang, "Development and validation of the Chinese version of the diabetes management self-efficacy scale," International Journal of Nursing Studies, vol. 45, no. 4, pp. 534-542, 2008.

[26] R. W. Brislin, "Back-translation for cross-cultural research," Journal of Cross-Cultural Psychology, vol. 1, pp. 185-216, 1970.

[27] L. Veneziano and J. Hooper, "A method for quantifying content validity of health-related questionnaires," American Journal of Health Behavior, vol. 21, no. 1, pp. 67-70, 1997.

[28] C. B. Terwee, S. D. Bot, M. R. de Boer et al., "Quality criteria were proposed for measurement properties of health status questionnaires," Journal of Clinical Epidemiology, vol. 60, no. 1, pp. 34-42, 2007.

[29] D. J. Toobert, S. E. Hampson, and R. E. Glasgow, “The summary of diabetes self-care activities measure: results from 7 studies and a revised scale," Diabetes Care, vol. 23, no. 7, pp. 943-950, 2000.

[30] S. Chang and M. Song, "The validation and reliability of a Korean version of the Summary of Diabetes Self-Care Activities questionnaire for older patients with type 2 diabetes," Korean Journal of Adult Nursing, vol. 21, pp. 235-244, 2009.

[31] E. J. Choi, M. Nam, S. H. Kim et al., "Psychometric properties of a Korean version of the summary of diabetes self-care activities measure," International Journal of Nursing Studies, vol. 48, no. 3 , pp. 333-337, 2011.

[32] H. F. Kaiser, "An index of factorial simplicity," Psychometrika, vol. 39, no. 1, pp. 31-36, 1974.

[33] B. G. Tabachnick and L. S. Fidell, Using Multivariate Statistics, Pearson Education, Upper Saddle River, NJ, USA, 6th edition, 2012.

[34] L.-T. Hu and P. M. Bentler, "Cutoff criteria for fit indexes in covariance structure analysis: conventional criteria versus new alternatives," Structural Equation Modeling, vol. 6, no. 1, pp. 155, 1999.

[35] R. B. Kline, Principles and Practice of Structural Equation Modeling, Guilford Press, New York, NY, USA, 2nd edition, 2005.

[36] R. C. MacCallum, M. W. Browne, and H. M. Sugawara, "Power analysis and determination of sample size for covariance structure modeling," Psychological Methods, vol. 1, no. 2, pp. 130-149, 1996.

[37] Korean Diabetes Association, Clinical Practice Guideline: Type 2 Diabetes, 2013, http://www.diabetes.or.kr/pro/publish/guide_ 2013.php? mode $=$ list.

[38] M. A. Pett, N. R. Lackey, and J. J. Sullivan, Make Sense of Factor Analysis, Sage, Thousand Oaks, Calif, USA, 2003.

[39] B. M. Byrne, Structural Equation Modeling with AMOS: Basic Concepts, Applications, and Programming, Taylor \& Francis, New York, NY, USA, 2nd edition, 2010.

[40] J. C. Nunnally and I. H. Bernstein, Psychometric Theory, McGraw-Hill, New York, NY, USA, 1994.

[41] P. Rapley, A. Passmore, and M. Phillips, "Review of the psychometric properties of the diabetes self-efficacy scale: Australian longitudinal study," Nursing \& Health Sciences, vol. 5, no. 4, pp. 289-297, 2003.

[42] J. D. Cook, S. J. Hepworth, and P. B. Warr, The Experience of Work, Academic Press, San Diego, Calif, USA, 1981.

[43] H. C. W. De Vet, H. J. Adèr, C. B. Terwee, and F. Pouwer, "Are factor analytical techniques used appropriately in the validation of health status questionnaires? A systematic review on the quality of factor analysis of the SF-36," Quality of Life Research, vol. 14, no. 5, pp. 1203-1218, 2005.

[44] L. G. Portney and M. P. Watkins, Foundations of Clinical Research. Applications to Practice, Pearson/Prentice Hall, Upper Saddle River, NJ, USA, 2009.

[45] P. M. Fayers and D. Machin, Quality of Life: The Assessment, Analysis and Interpretation of Patient-Related Outcomes, John Wiley \& Sons, West Sussex, UK, 2nd edition, 2007.

[46] R. G. Marx, A. Menezes, L. Horovitz, E. C. Jones, and R. F. Warren, "A comparison of two time intervals for test-retest reliability of health status instruments," Journal of Clinical Epidemiology, vol. 56, no. 8, pp. 730-735, 2003. 


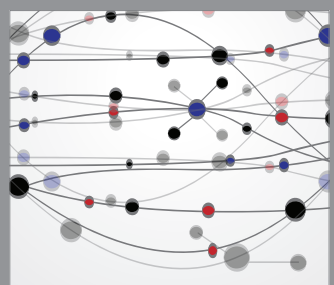

The Scientific World Journal
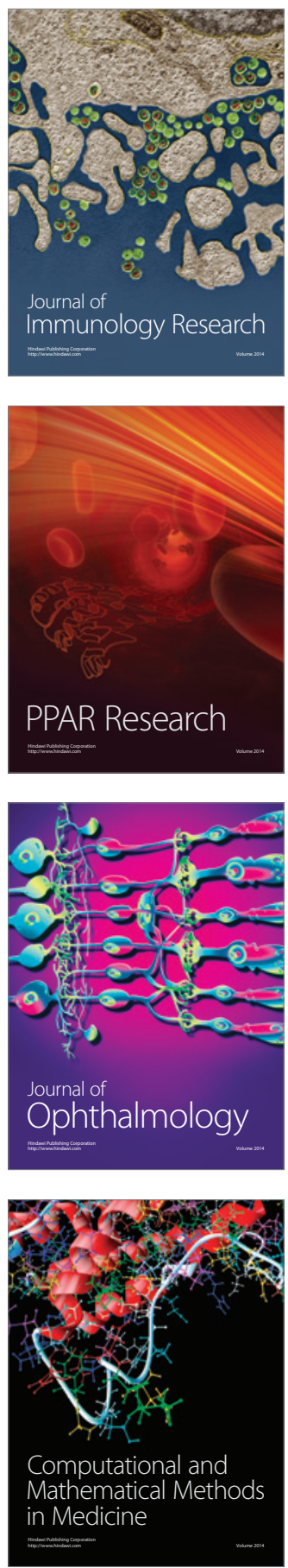

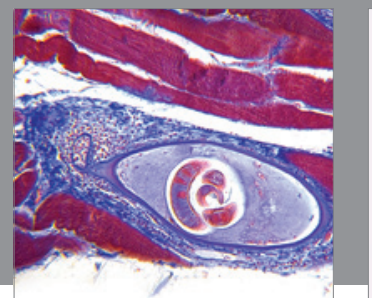

Gastroenterology

Research and Practice
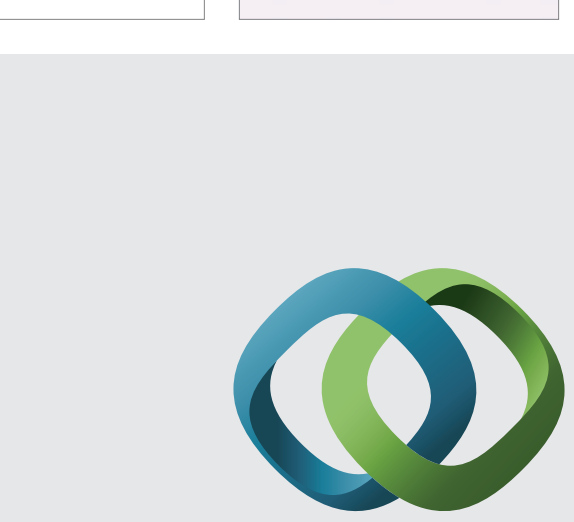

\section{Hindawi}

Submit your manuscripts at

http://www.hindawi.com
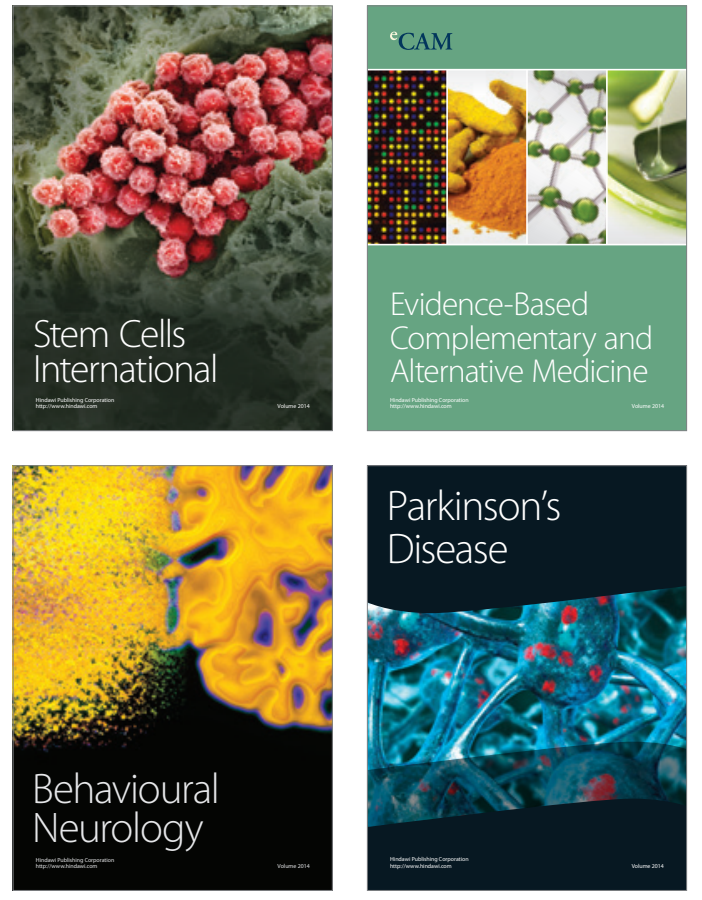
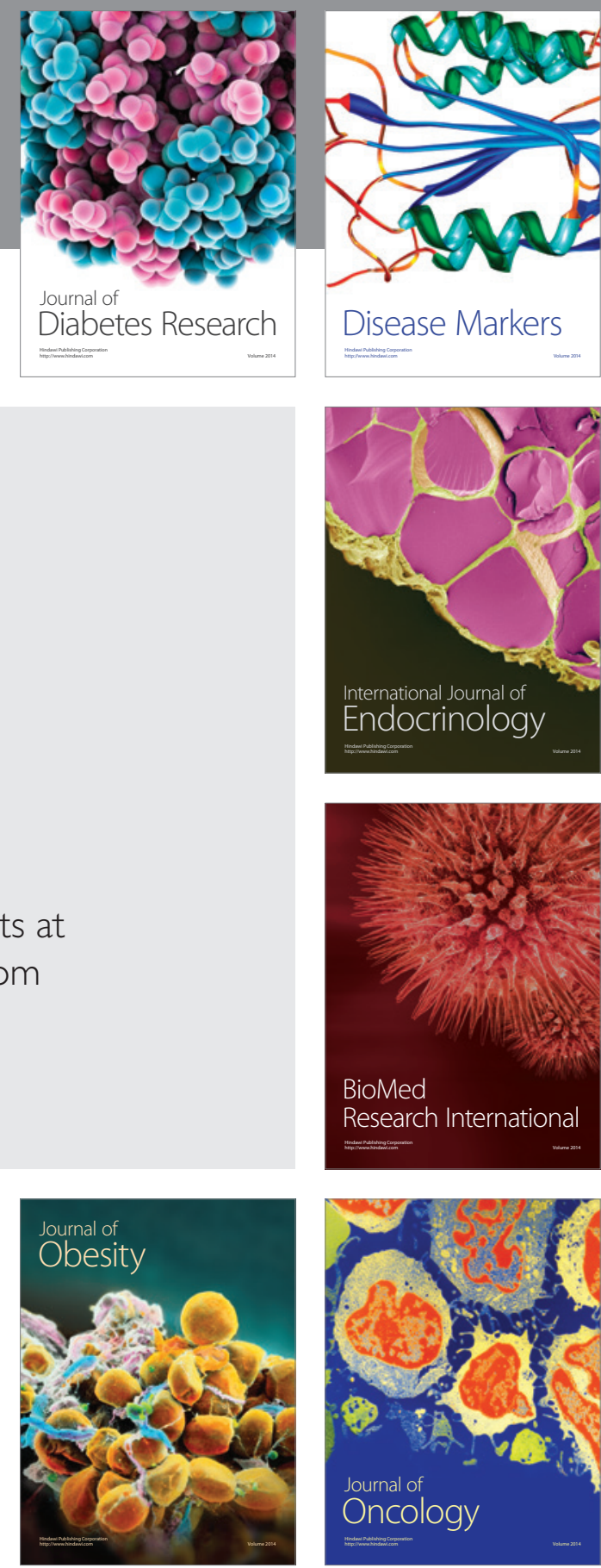

Disease Markers
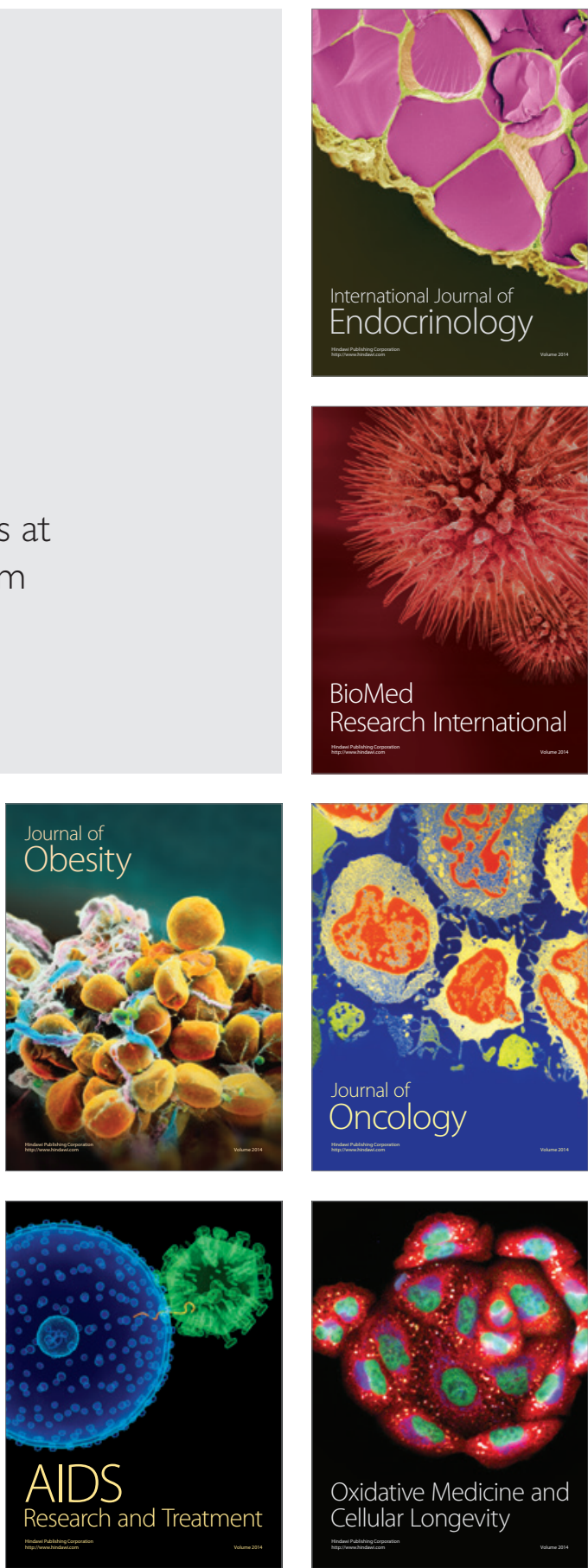